\title{
PERSPECTIVES IN OPTOELECTRONICS
}

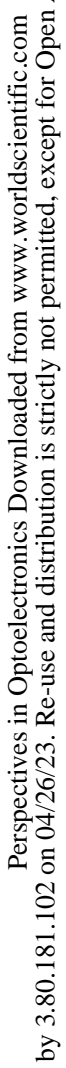


This page is intentionally left blank

\section{This page is intentionally left blank}




\section{PERSPECTIVES IN}

\section{OPTOELECTRONICS}

Editor

\section{Sudhanshu S. Jha}

Distinguished Professor

Tata Institute of Fundamental Research

Bombay, India

Honorary Professor

Jawaharlal Nehru Centre for Advanced Scientific Research

Bangalore, India 
Published by

World Scientific Publishing Co. Pte. Ltd.

P O Box 128, Farrer Road, Singapore 9128

USA office: Suite 1B, 1060 Main Street, River Edge, NJ 07661

UK office: 57 Shelton Street, Covent Garden, London WC2H 9HE

\section{Library of Congress Cataloging-in-Publication Data}

Perspectives in optoelectronics / editor, Sudhanshu S. Jha.

p. $\mathrm{cm}$.

Includes bibliographical references and index.

ISBN 9810220227 (hardcover)

1. Optoelectronics. I. Jha, Sudhanshu S. (Sudhanshu Shekhar), 1940

TA1750.P43 1995

$621.36--d c 20$

95-37189

CIP

\section{British Library Cataloguing-in-Publication Data}

A catalogue record for this book is available from the British Library.

Copyright () 1995 by World Scientific Publishing Co. Pte. Ltd.

All rights reserved. This book, or parts thereof, may not be reproduced in any form or by any means, electronic or mechanical, including photocopying, recording or any information storage and retrieval system now known or to be invented, without written permission from the Publisher.

For photocopying of material in this volume, please pay a copying fee through the Copyright Clearance Center, Inc., 222 Rosewood Drive, Danvers, Massachusetts 01923, USA. 


\section{TO ALL OUR TEACHERS}

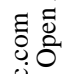

巳

氞

음

D

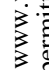

छิ응

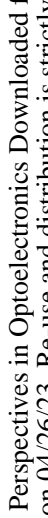

ชิ

0
$\dot{0}$
$\dot{0}$
$\infty$
$\dot{0}$
$\dot{2}$ 
This page is intentionally left blank

\section{This page is intentionally left blank}




\section{PREFACE}

In recent years, optoelectronics has emerged as a rapidly expanding frontier area of research and development. This field has, of course, crucial overlaps with optics, materials science, semiconductor technology and microelectronics. Important developments in all these areas and rapid strides made in materialgrowth and device-fabrication technologies, are indeed responsible for the steady progress of optoelectronics. With the availability of a wide range of tunable, pulsed, coherent optical sources and amplifiers, the low-loss single-mode silica-fiber optical transmission medium, sensitive photodetectors, novel ultra-fast modulation techniques, and efficient nonlinear optical materials, optoelectronics is playing a major role in both applied as well as basic sciences. In years to come, it is destined to change the face of information technology and robotics, involving optical sensing and control, information storage, signal and image processing, communications, and computing. Because of the possibility of using large bandwidths available at optical frequencies, and the two-dimensional optical processing, the conventional microelectronics is moving progressively towards the new technology of optoelectronics and photonics. The idea is to use and integrate in real systems more and more photonic devices in which optical photons instead of electronic charge-carriers are manipulated for performing the desired task.

In addition to a large number of review articles and monographs, several good books dealing with different aspects of optoelectronics have already been published. However, for some time now a need for a comprehensive volume describing most of the current developments and future directions in the field has been felt by many research workers and students of the field. Although, this task is necessarily difficult because of the vast spread of the subject, the present book represents an attempt in this direction. This volume tries to cover a substantial number of topics of recent importance in the field as well as topics with high potential for future development. Apart from the inclusion of crucial and useful details of lasting importance in each chapter of the volume, dealing with different aspects of the field, the idea is to present an overall view of the developments in their true relationship, i.e. a perspective. Starting from simple concepts, each chapter contains an extensive discussion of most of the recent developments in the area. Authors of these chapters are experts on respective topics. The level of each presentation is such that the volume will be useful to $\mathrm{Ph}$.D. graduate students as well as to other researchers in the field.

The volume consists of 16 chapters. The first one is an introductory chapter which describes the nature and scope of optoelectronics, and some of the basic principles of optics relevant to the field. As optical sources suitable for various applications, semiconductor lasers and optical amplifiers are discussed in Chapter 2. In the context of specific applications to optical communications, erbium-doped fiber amplifiers are considered in greater detail in Chapter $\mathbf{9}$ on lightwave telecommunications. A very comprehensive exposition of optical transmission through optical fibers is 
given in Chapter 5, whereas Chapter $\mathbf{6}$ deals with different kinds of photodetectors. Two important applications related to optical processing and optical computing are discussed in Chapters $\mathbf{7}$ and $\mathbf{8}$, respectively. A review of the current status of optoelectronic and photonic integrated circuits in the context of integrated receivers and transmitters in lightwave communication systems is presented in Chapter $\mathbf{4}$, whereas a more general discussion of guided-wave theory and integrated optics is given in Chapter 12. Chapter $\mathbf{1 3}$ deals with a very useful area of optical interconnections. The field of optical metrology and various developments in optical sensor technology are discussed in Chapter 14. An interesting development in ultrafast optoelectronics related to optical generation of electromagnetic pulses with terahertz bandwidths is described in Chapter 3.

The field of nonlinear optics and efficient materials for fabricating nonlinear optical devices are covered in Chapters $\mathbf{1 0}$ and 11, respectively. The role of sensitive quantum optical detectors for gravitational waves and precision optical measurements for testing the foundations of physics are presented very briefly in Chapter 15, whereas Chapter 16 describes in a great detail various concepts in the theory of optical diffraction, dispersion and space-time Fourier optics, useful for optical image processing.

Because of different types of constraints, including the size of the volume, I must admit that we have not been able to cover adequately some other important topics in the field. The notable omissions include a comprehensive review of the area on optical storage, memory and holography, besides other topics dealing with very important applications to basic sciences and medicine, and to sectors like energy and defence. Inspite of this, I hope the volume has its own character, and it will be of interest to a large number of scientists and engineers.

I shall like to take this opportunity to express my sincere thanks and appreciation to all the contributors of the volume. This has been a great experience for me, and I am extremely grateful to all the authors for their excellent co-operation. In addition, I am very much obliged to Dr. K.C. Rustagi of the Centre for Advanced Technology, Indore, for his help in careful checking of most of the manuscripts. I shall also like to thank several members of the Tata Institute of Fundamental Research, Bombay, who helped me to complete the task. In particular, I appreciate greatly the support provided by Mr. M.R. Shinde, and also by Mr. R.N. Bathija and Mr. R.S. Pawar. Finally, it is a great pleasure for me to thank Mr. Tony Moore, Editor, World Scientific Publishing Company, London, who initiated the whole idea about this volume, and Dr.(Ms.) Anju Goel, the Scientific Editor, World Scientific, Singapore, for their continued cooperation and support in bringing out the volume in time. 


\section{CONTRIBUTORS}

M.A.G. Abushagur, University of Alabama, Huntsville, Alabama, USA

R.J. Berinato, Dynetics Inc., Huntsville, Alabama, USA

D.N. Bose, Indian Institute of Technology, Kharagpur, India

V.B. Braginsky, Moscow State University, Moscow, Russia

H.J. Caulfield, Alabama A\&M University, Normal, Alabama, USA

S. Chandrasekhar, AT\&T Bell Laboratories, Holmdel, New Jersey, USA

B. Colombeau, Université de Limoges, Limoges, France

N.K. Dutta, AT\&T Bell Laboratories, Murray Hill, New Jersey, USA

C. Froehly, Université de Limoges, Limoges, France

C.R. Giles, AT\&T Bell Laboratories, Holmdel, New Jersey, USA

G.L. Henderson, Naval Surface Warfare Centre, Panama City, Florida, USA

S.S. Jha, Tata Institute of Fundamental Research, Bombay, India

Y. Jin, Rensselaer Polytechnic Institute, Troy, New York, USA

J. Kinser, Alabama A\&M University, Normal, Alabama, USA

P.J.R. Laybourn, University of Glasgow, Glasgow, Scotland

G. Lebreton, GESSY, Université de Toulon, La Garde, France

T. Li, AT\&T Bell Laboratories, Holmdel, New Jersey, USA

P.C. Mehta, Instruments Research \& Development Estt., Dehra Dun, India

A.G. Mignani, IROE-CNR, Firenze, Italy

B.P. Pal, Indian Institute of Technology Delhi, New Delhi, India

M.A. Pollack, AT\&T Bell Laboratories, Holmdel, New Jersey, USA

P.N. Prasad, State University of New York, Buffalo, New York, USA

G.C. Righini, IROE-CNR, Firenze, Italy

K.C. Rustagi, Centre for Advanced Technology, Indore, India

M. Vampouille, Université de Limoges, Limoges, France

W.M.K.P. Wijekoon, State University of New York, Buffalo, New York, USA

X.-C. Zhang, Rensselaer Polytechnic Institute, Troy, New York, USA 
This page is intentionally left blank

\section{This page is intentionally left blank}




\section{CONTENTS}

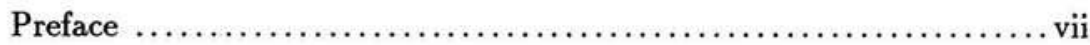

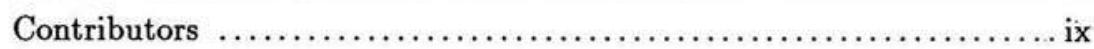

\section{CHAPTER 1 \\ INTRODUCTION}

Sudhanshu S. Jha

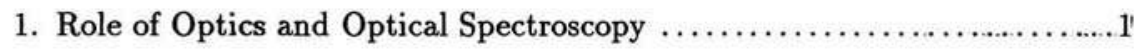

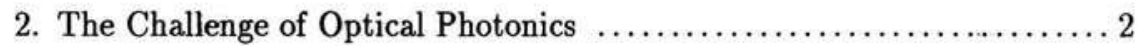

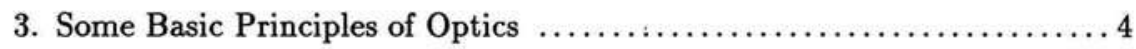

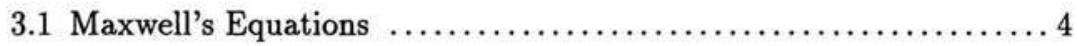

3.2 Linear Optics : Propagation and Dispersion $\ldots \ldots \ldots \ldots \ldots \ldots \ldots 6$

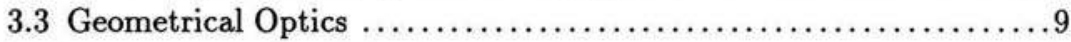

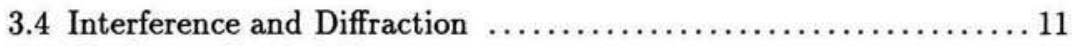

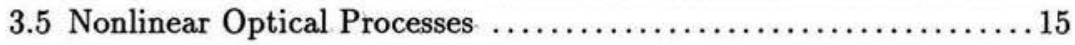

3.6 Quantized Fields and Photon States $\ldots \ldots \ldots \ldots \ldots \ldots \ldots \ldots \ldots \ldots$

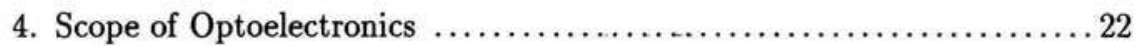

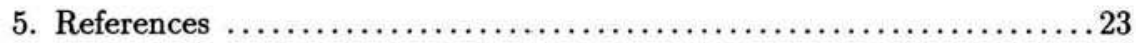

\section{CHAPTER 2 \\ SEMICONDUCTOR LASERS AND OPTICAL AMPLIFIERS N. K. Dutta}

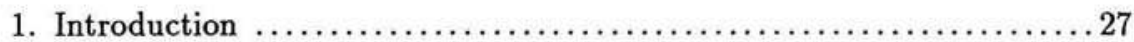

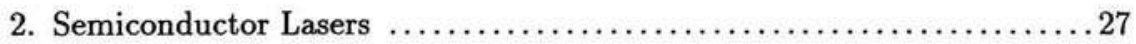

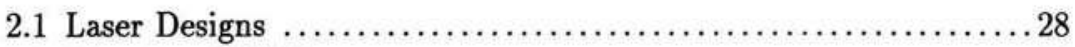

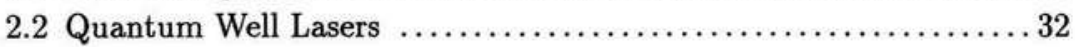

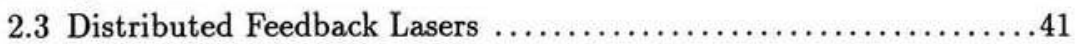

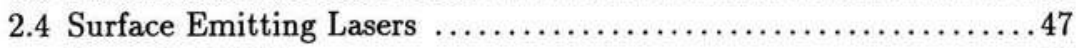

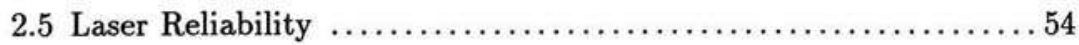

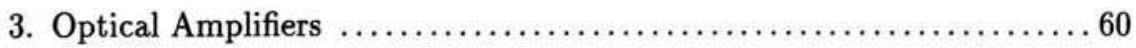

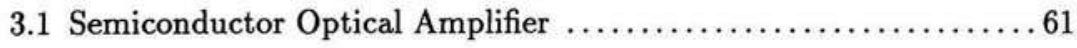


3.2 Fiber Amplifiers

4. References

\section{CHAPTER 3}

\section{OPTICALLY GENERATED THz BEAMS FROM DIELECTRICS} X.-C. Zhang and Y. Jin

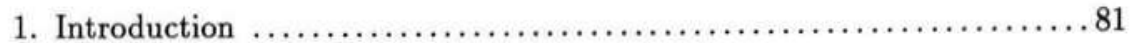

2. Large-Aperture Photoconducting Antennas $\ldots \ldots \ldots \ldots \ldots \ldots \ldots \ldots \ldots \ldots$

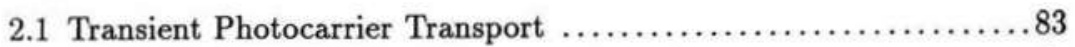

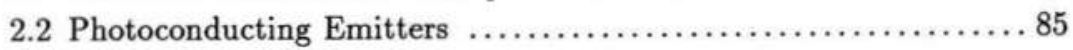

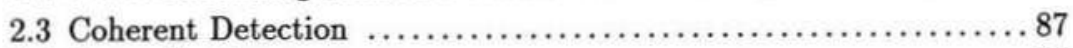

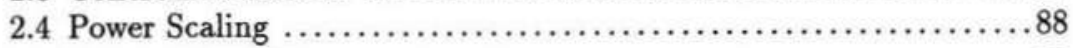

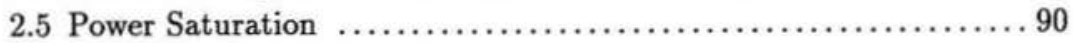

3. $\mathrm{THz}$ Radiation from Semiconductor Interfaces $\ldots \ldots \ldots \ldots \ldots \ldots \ldots . \ldots 9$

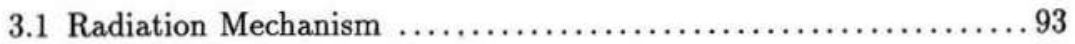

3.2 Electric Dipole Approximation $\ldots \ldots \ldots \ldots \ldots \ldots \ldots \ldots \ldots \ldots \ldots . \ldots \ldots$

3.3 Emission from a Semiconductor Depletion Field $\ldots \ldots \ldots \ldots \ldots \ldots 96$

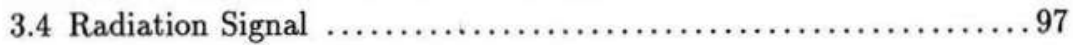

3.5 Transient Carriers in a Magnetic Field $\ldots \ldots \ldots \ldots \ldots \ldots \ldots \ldots . \ldots . \ldots 9$

3.6 Magnetic Switching of $\mathrm{THz}$ Beams ....................... 103

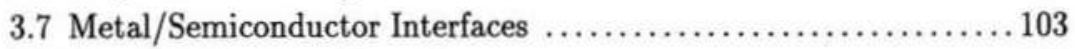

3.8 Pump-Probe-Gate Experiments ......................... 105

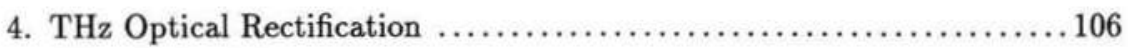

4.1 Historical Background of Optical Rectification $\ldots \ldots \ldots \ldots \ldots \ldots 106$

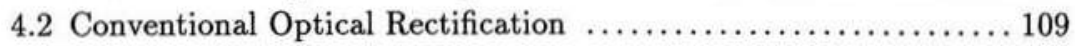

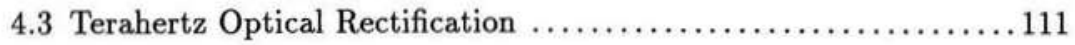

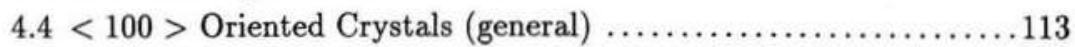

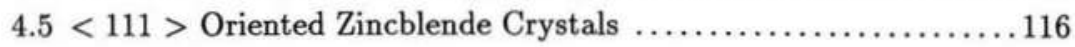

$4.6<110>$ Oriented Zincblende Crystals . . . . . . . . . . . . . . . 117

$4.7 \mathrm{THz}$ Optical Rectification from Nonlinear Crystals ............ 118

4.8 Resonant Second Order Nonlinear Susceptibility .............. 125

4.9 Difference-frequency Generation and Sum-frequency Generation .130

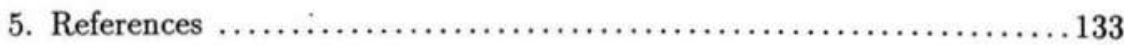


OPTOELECTRONIC AND PHOTONIC INTEGRATED CIRCUITS S. Chandrasekhar and Martin A. Pollack

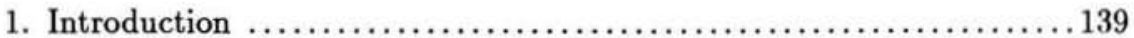

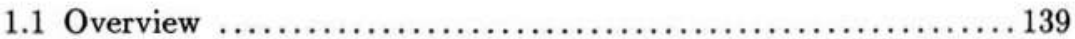

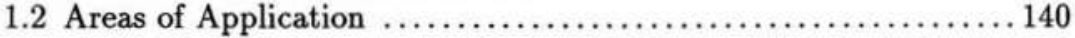

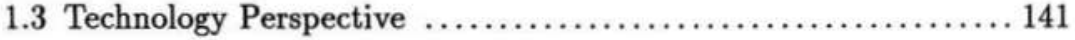

2. Optoelectronic Integrated Circuits (OEICs) $\ldots \ldots \ldots \ldots \ldots \ldots \ldots \ldots \ldots \ldots \ldots \ldots \ldots \ldots$

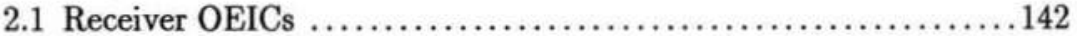

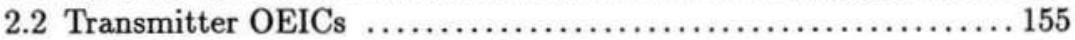

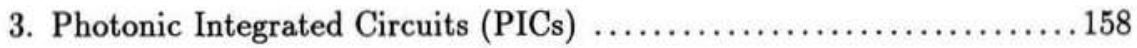

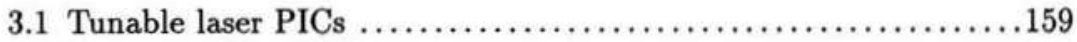

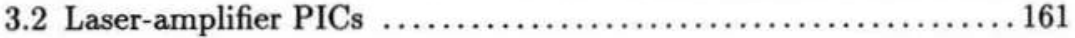

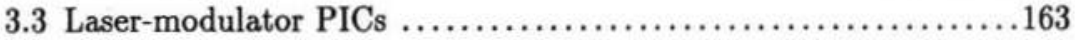

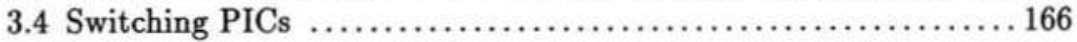

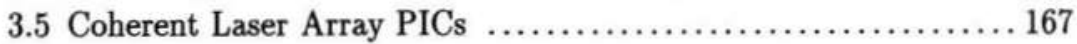

3.6 Multi-wavelength Source Array PICs ....................... 169

3.7 Grating and Wavelength Router-based PICs ................ 172

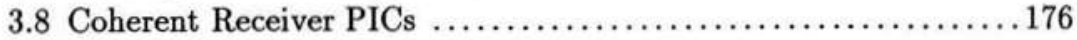

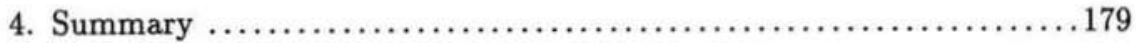

4.1 Trends in Research ................................... 179

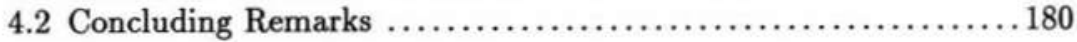

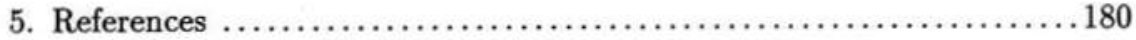

\section{CHAPTER 5 \\ OPTICAL TRANSMISSION : FIBER OPTICS Bishnu P. Pal}

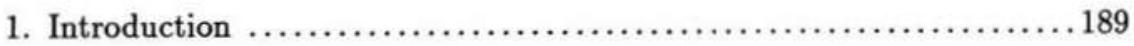

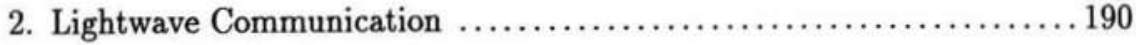

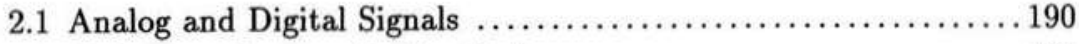

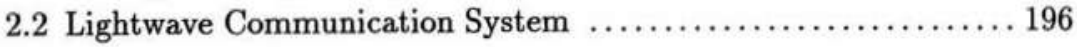

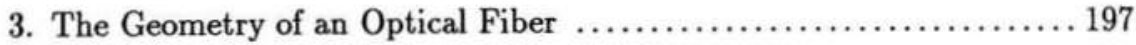

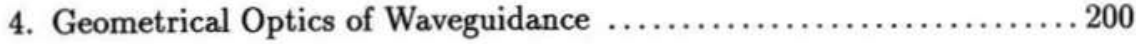


4.1 Step-index Fiber and Concept of Numerical Aperture .........200

4.2 Temporal Pulse Dispersion in a Step-index Fiber ............. 202

4.3 Ray Paths in a Graded Index Fiber and Temporal Dispersion in a Parabolic Index Fiber ............................... 204

4.4 Temporal Dispersion in a Graded-index Multimode Fiber ...... 207

5. Electromagnetic Analysis of Propagation in Optical Fibers ..........209

5.1 Basic Framework for Guided Wave Optics in Step-index Fibers ..209

5.2 Mode cut-off and Single-mode Operating regime $\ldots \ldots \ldots \ldots \ldots 214$

5.3 Power Flow in the Core and the Cladding $\ldots \ldots \ldots \ldots \ldots \ldots \ldots \ldots \ldots \ldots \ldots$

5.4 Electromagnetics of Multimode Graded Index Fibers ............218

5.5 Modal Spacing and N.A. of Graded Index Fiber ............. 220

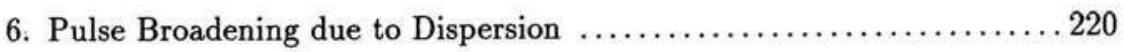

6.1 Temporal Dispersion in a Multimode Fiber $\ldots \ldots \ldots \ldots \ldots \ldots \ldots \ldots \ldots \ldots \ldots$

6.2 Temporal Dispersion in a Single-mode Fiber . ................224

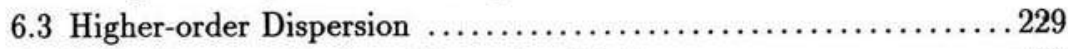

6.4 Pulse Propagation in a Dispersive Medium ................ 229

6.5 Pulse Chirping due to Dispersion . ......................... 232

6.6 Pulse Compression of a Chirped Pulse ...................233

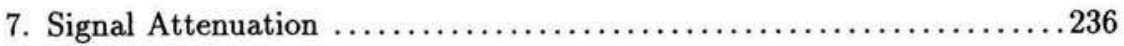

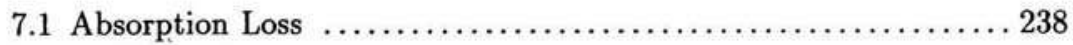

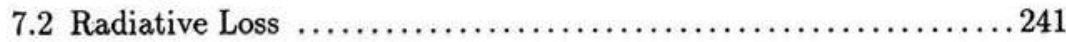

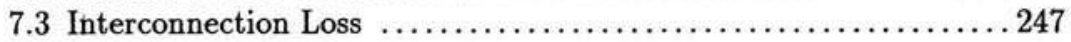

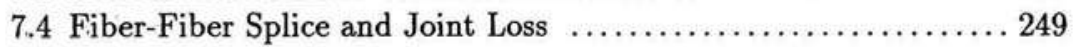

8. Important Characteristics of Single-Mode Fibers $\ldots \ldots \ldots \ldots \ldots \ldots \ldots 253$

8.1 Mode Field Profile and Mode Field Radius ............... 253

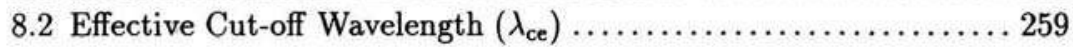

9. Advanced Single-mode Fibers : Design Issues ..................261

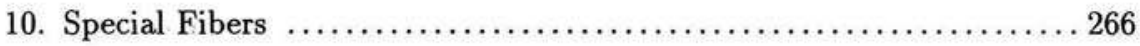

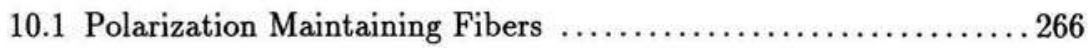

10.2 Truly Single Polarization Single-Mode (SPSM) Fibers . . . . . . . 269

11. Nonlinear Optical Effects in Fiber Transmission ................ 271

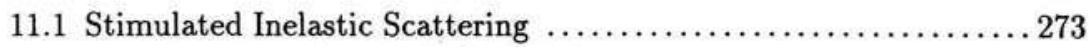

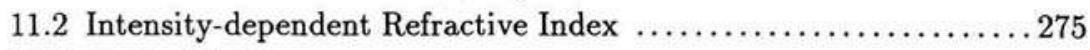

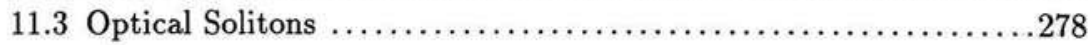




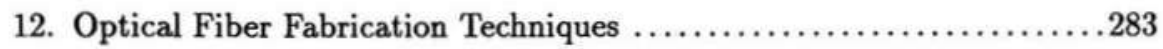

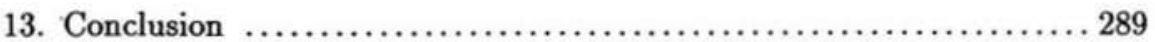

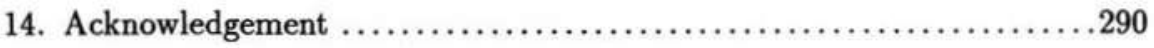

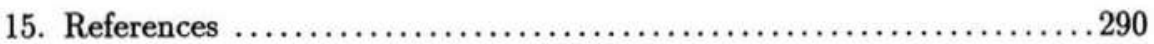

\section{CHAPTER 6 PHOTODETECTORS D. N. Bose}

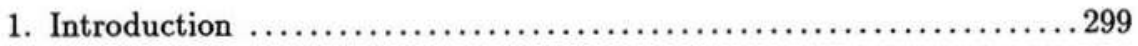

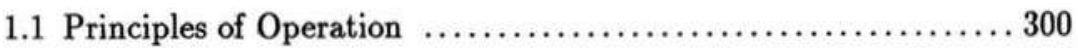

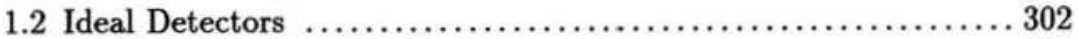

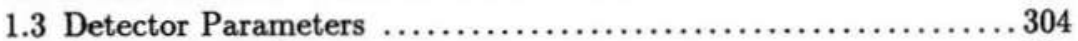

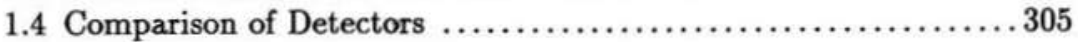

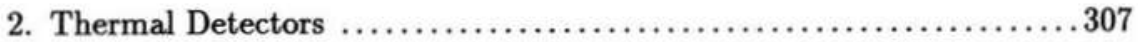

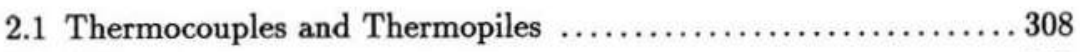

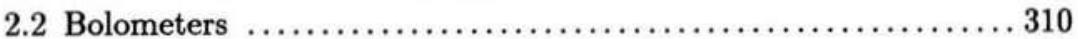

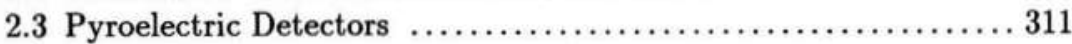

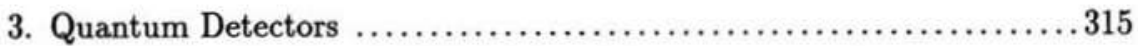

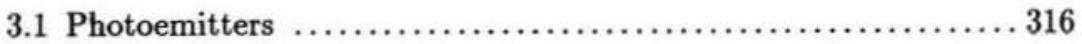

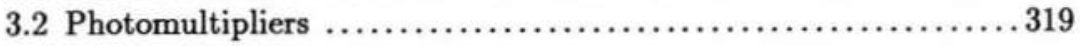

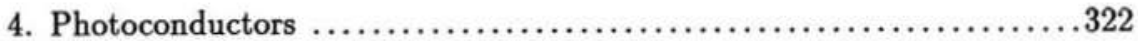

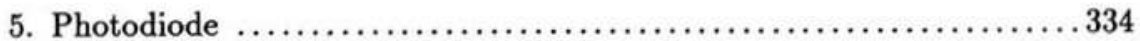

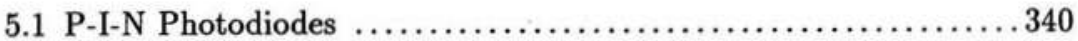

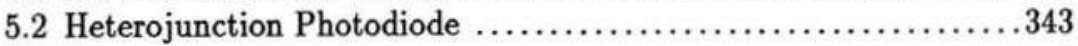

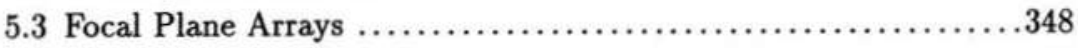

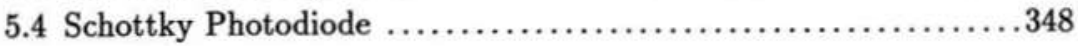

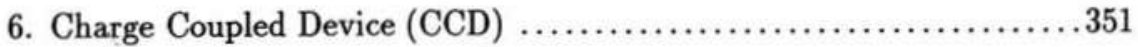

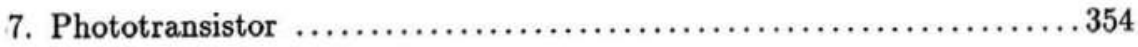

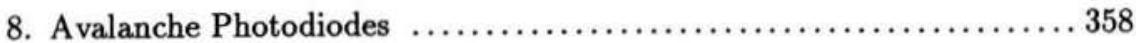

8.1 Resonant Cavity Photodiodes ........................... 371

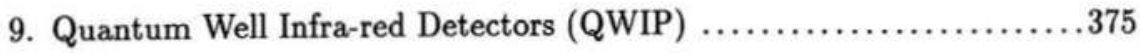

10. Conclusions and Future Developments ....................... 380 


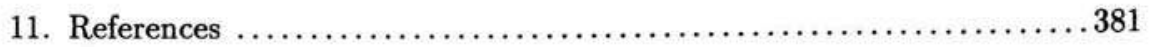

\section{CHAPTER 7}

\section{OPTICAL SIGNAL AND IMAGE PROCESSING M.A.G. Abushagur, H.J. Caulfield, J. Kinser, R.J. Berinato and G.L. Henderson}

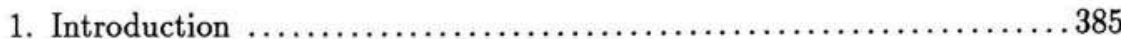

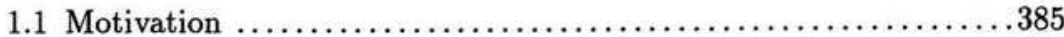

1.2 One-Dimensional vs. Two-Dimensional ................... 389

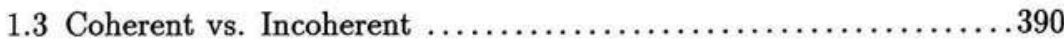

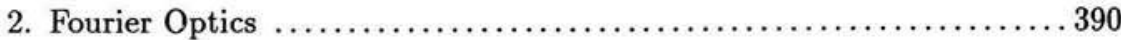

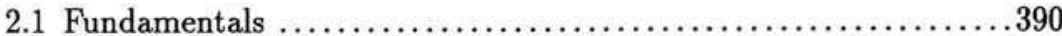

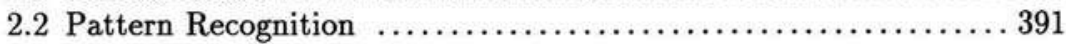

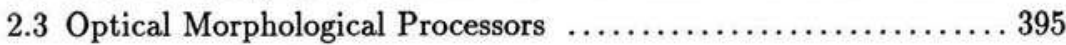

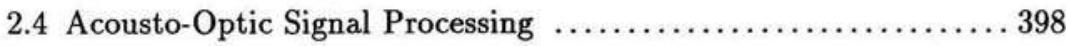

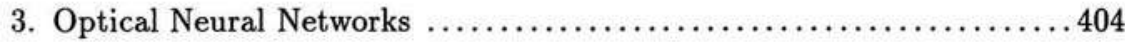

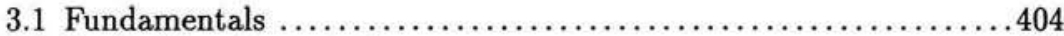

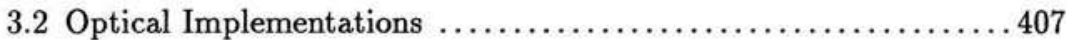

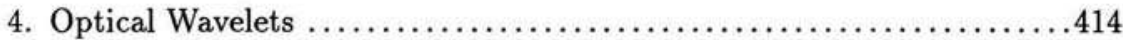

4.1 Wavelet Transforms ................................... 414

4.2 Optical Implementation of Wavelet Transforms $\ldots \ldots \ldots \ldots \ldots \ldots 415$

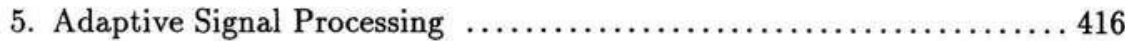

5.1 Time-Based Algorithms .............................. 417

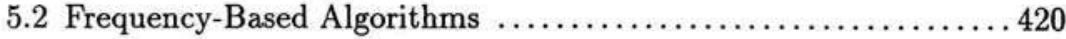

5.3 Optical Adaptive Filter Architectures $\ldots \ldots \ldots \ldots \ldots \ldots \ldots \ldots . \ldots 23$

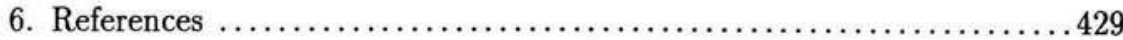

\section{CHAPTER 8}

\section{SPATIAL LIGHT MODULATORS AND OPTICAL COMPUTING Guy Lebreton}

1. Introduction: The History of Optical Computing $\ldots \ldots \ldots \ldots \ldots . \ldots 49$

1.1 Transversal Filtering Architectures 
1.2 Parallel Analog Computing Architectures ..................441

1.3 Parallel Digital Computing Architectures ................. 442

1.4 Parallel Logic Arrays ...................................443

1.5 Parallel Optical Logic and Arithmetic Operations ............445

2. Spatial Light Modulators using Solid Materials ............... 447

2.1 Acousto-optic Spatial Light Modulators ................. 447

2.2 Electro-optic and other Solid-material Spatial Light Modulators .457

3. Spatial Light Modulators using Liquid Crystals ................. 468

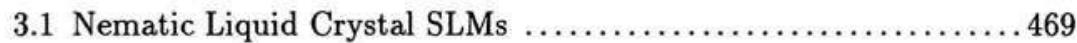

3.2 Ferroelectric Liquid Crystal SLMs $\ldots \ldots \ldots \ldots \ldots \ldots \ldots \ldots \ldots \ldots 44$

3.3 Smart-pixels Liquid Crystal SLMs $\ldots \ldots \ldots \ldots \ldots \ldots \ldots \ldots \ldots \ldots 79$

4. Spatial Light Modulators using Semiconductors ................480

4.1 Nonlinear Electrooptic Effects in Semiconductors ............ 481

4.2 Optical Bistability with Semiconductors $\ldots \ldots \ldots \ldots \ldots \ldots \ldots \ldots . \ldots 45$

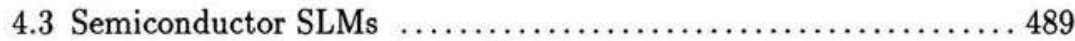

4.4 Smart-pixels Semiconductor SLMs $\ldots \ldots \ldots \ldots \ldots \ldots \ldots \ldots \ldots \ldots 7$

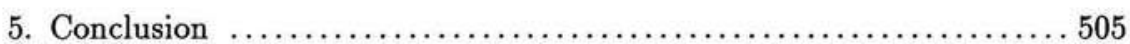

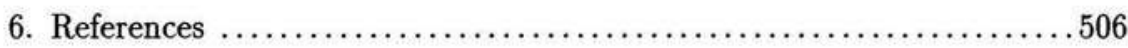

\section{CHAPTER 9 \\ OPTICAL AMPLIFIERS IN LIGHTWAVE TELECOMMUNICATIONS \\ Tingye Li and C. Randy Giles}

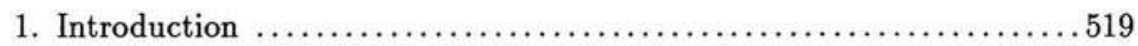

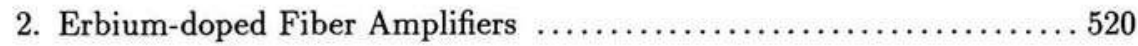

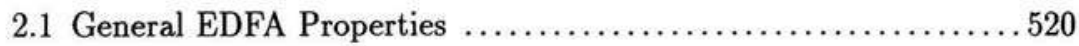

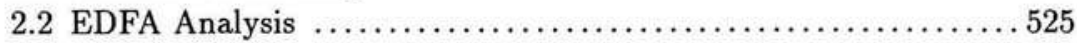

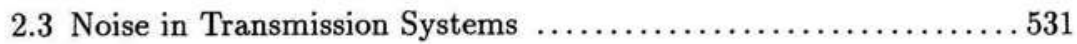

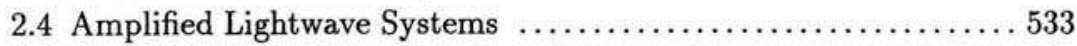

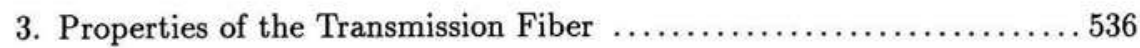

4. Undersea-Cable Transmission Systems ...................... 543

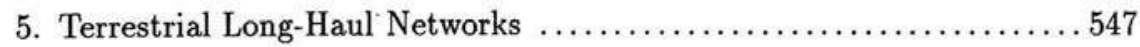




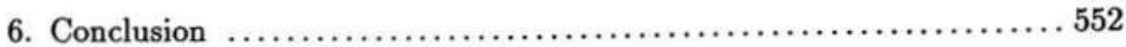

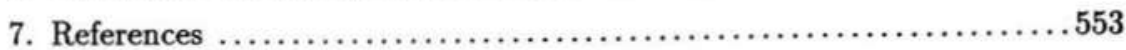

\section{CHAPTER 10 \\ NONLINEAR OPTICS \\ Kailash C. Rustagi}

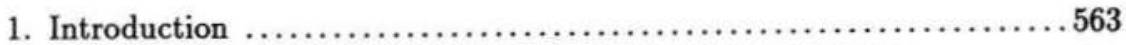

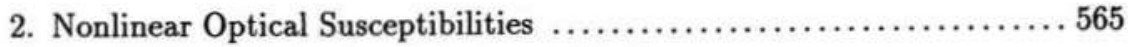

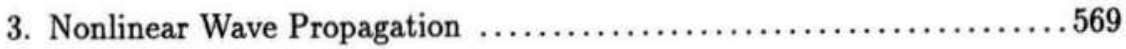

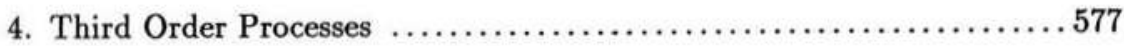

4.1 Optical Bistability .................................. 580

4.2 Degenerate Four Wave Mixing for Optical Phase Conjugation ...582

4.3 Wavefront Recovery by Optical Phase Conjugation ........... 583

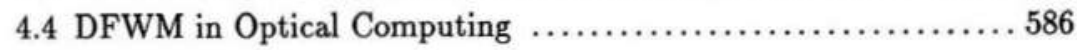

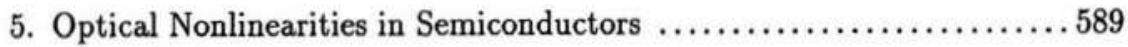

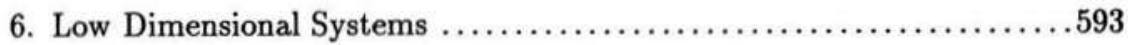

6.1 Quantum Wells .................................. 593

6.2 Semiconductor Doped Glasses ......................... 594

6.3 Metal Particles ..................................... 595

$6.4 \pi$-electron Systems .................................... 597

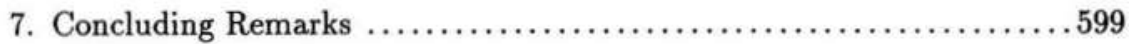

8. Acknowledgements ...................................... 599

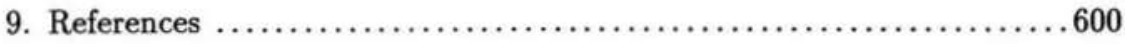

CHAPTER 11

MATERIALS FOR NONLINEAR OPTICS

W. M. K. P. Wijekoon and P. N. Prasad

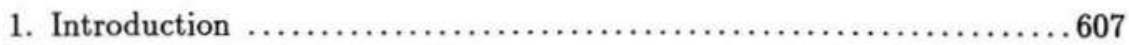

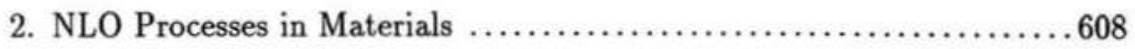

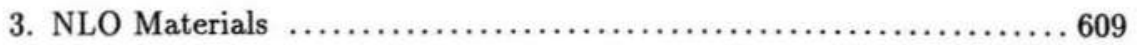




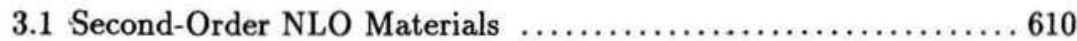

3.2 Measurement of $\beta$ : Electric Field Induced SHG $\ldots \ldots \ldots \ldots \ldots \ldots 12$

3.3 Measurement of $\chi^{(2)}$ : Crystals and Powders $\ldots \ldots \ldots \ldots \ldots \ldots 618$

3.4 Poled Materials .........................................628

3.5 Langmuir-Blodgett and Self-Assembled Monolayers ..........661

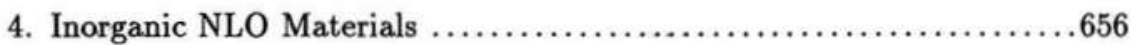

4.1 Ammonium Dihydrogenphosphate $\left(\mathrm{NH}_{4} \mathrm{H}_{2} \mathrm{PO}_{4}\right) \ldots \ldots \ldots \ldots \ldots 68$

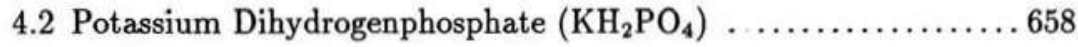

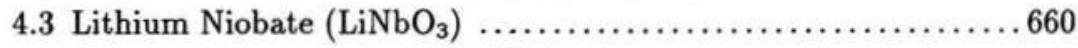

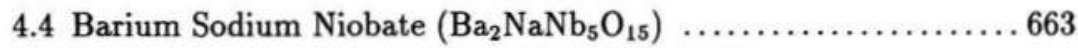

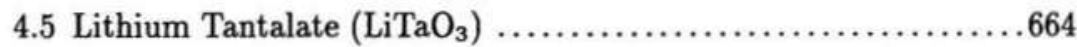

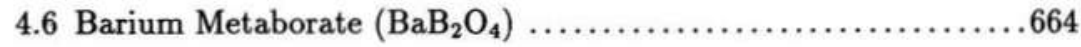

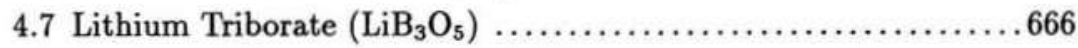

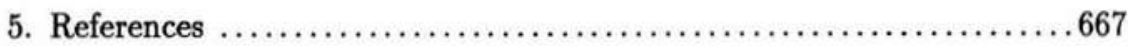

\section{CHAPTER 12 \\ INTEGRATED OPTICS \\ Giancarlo C. Righini and Peter J.R. Laybourn}

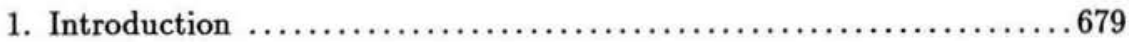

2. Guided-Wave Theory: Basic Concepts .....................680

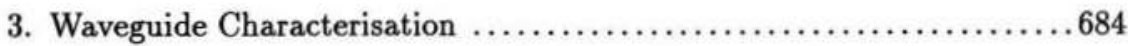

3.1 M-line and Dark-line Spectroscopy .......................6 684

3.2 Measurements of Losses and Other Parameters .............. 687

3.3 Photothermal Deflection Technique ........................692

4. Semiconductor Devices and Integrated Optoelectronics ............693

4.1 III-V Semiconductors as Integrated Optical Materials .........693

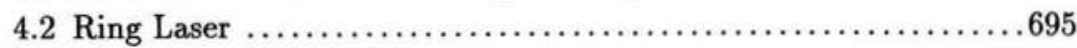

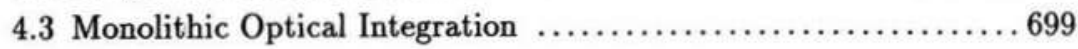

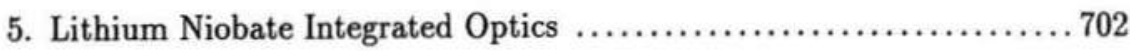

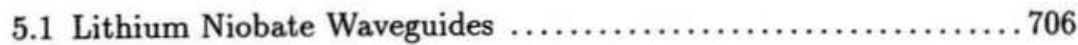

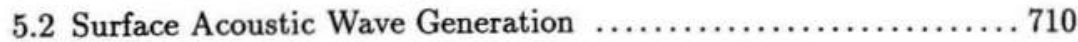

5.3 Integrated Optical Signal Processing Devices ............... 713 
6. Glass Integrated Optics

6.1 Ion-Exchanged Waveguides and Components ............... 715

6.2 High-Silica on Silicon Waveguides ........................ 719

6.3 Nonlinear and Active Glass Waveguides ................... 721

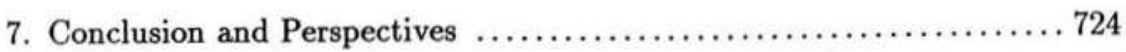

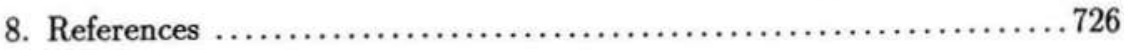

\section{CHAPTER 13 \\ OPTICAL INTERCONNECTS \\ P. C. Mehta}

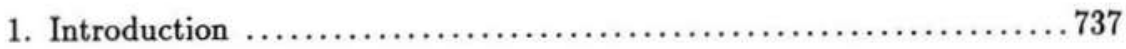

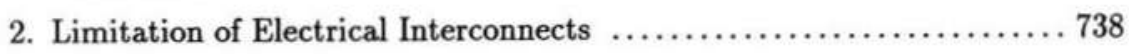

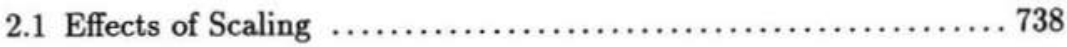

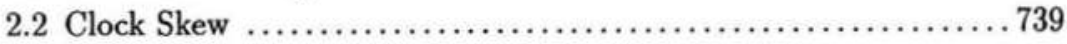

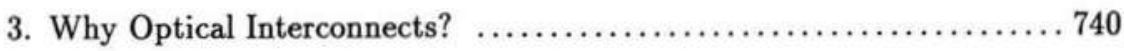

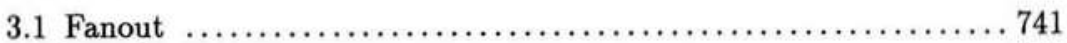

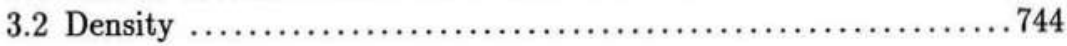

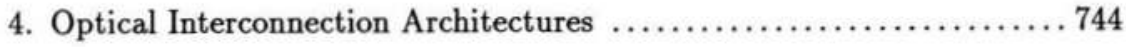

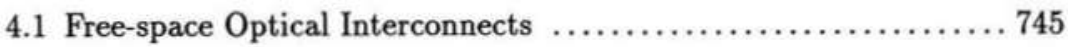

4.2 Holographic Optical Interconnects ........................ 749

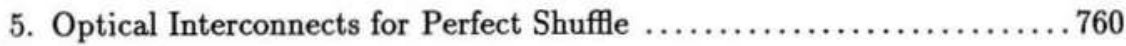

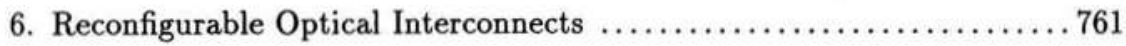

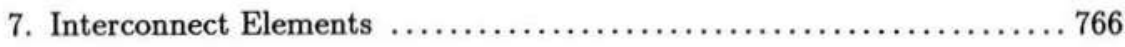

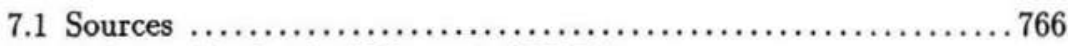

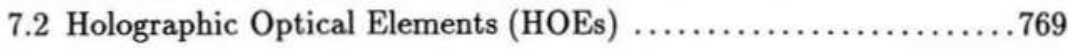

8. Applications of Optical Interconnects ..................... 774

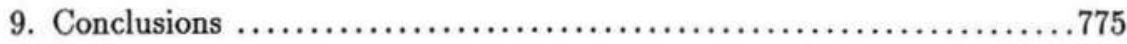

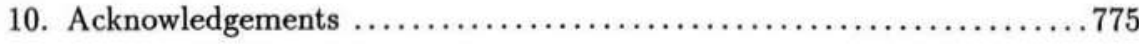

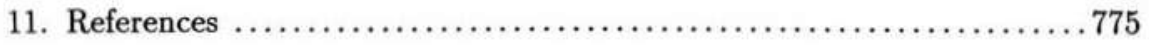




\section{OPTICAL METROLOGY AND SENSING Giancarlo C. Righini and Anna Grazia Mignani}

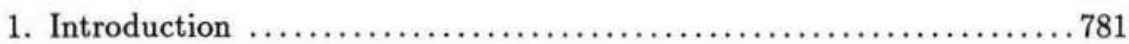

2. Position and Dimensional Measurements ...................... 782

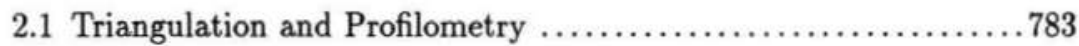

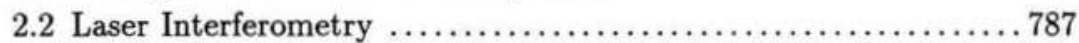

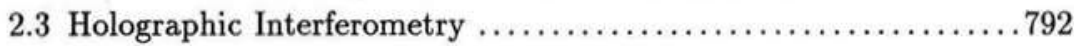

3. Other Optical Techniques for Metrology and Sensing $\ldots \ldots \ldots \ldots . \ldots 794$

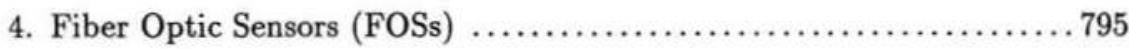

4.1 Why Use Them? ................................... 795

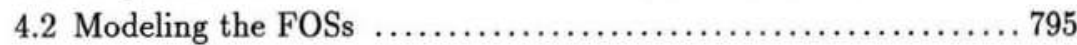

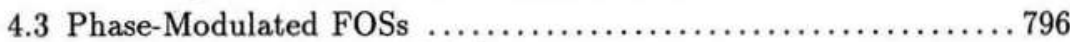

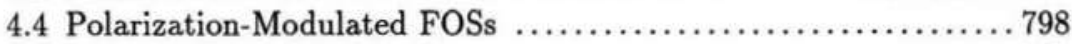

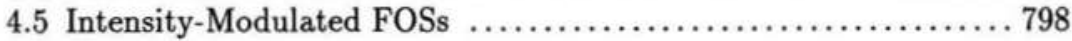

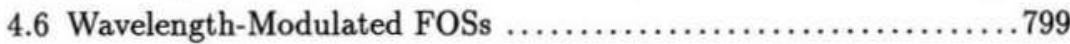

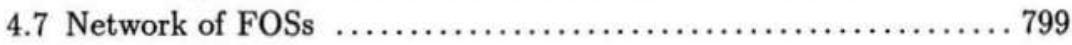

5. Advances in Fiber Optic Environmental Monitoring $\ldots \ldots \ldots \ldots \ldots 801$

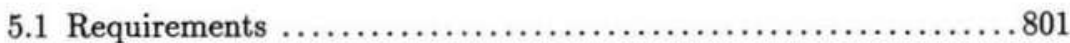

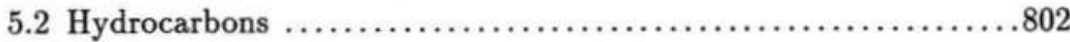

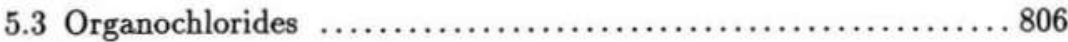

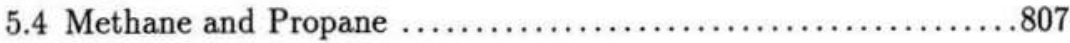

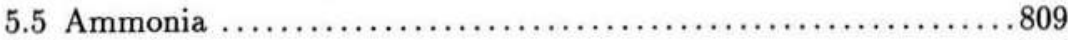

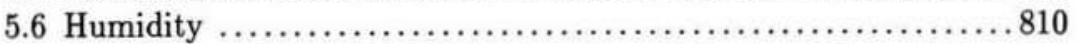

$5.7 \mathrm{pH}$ and Carbon Dioxide Partial Pressure of Sea- and Ocean Water 812

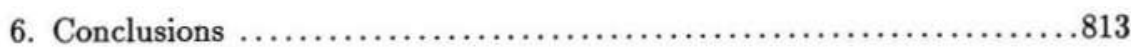

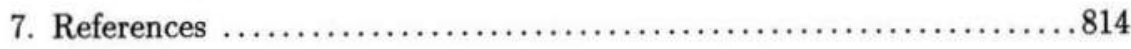

CHAPTER 15

QUANTUM OPTICS AND FOUNDATIONS OF PHYSICS

V. B. Braginsky

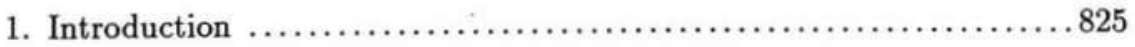


2. Reserves in Sensitivity and Resolution in Existing Quantum-Optical Methods

3. Prospects of New Methods in Quantum Optics

4. Possible Future Applications of Quantum-Optical Methods in Search

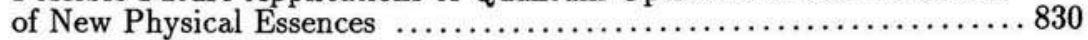

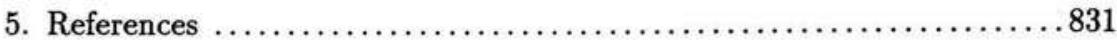

CHAPTER 16

DIFFRACTION, DISPERSION AND SPACE-TIME FOURIER OPTICS

C. Froehly, B. Colombeau and M. Vampouille

1. Paraxial Scalar Diffraction and Dispersion of Light Signals .........835

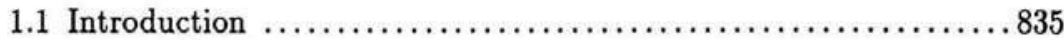

1.2 Paraxial Scalar Diffraction of Monochromatic Beams ..........836

1.3 Dispersion of Narrow Bandwidth Temporal Signals ..........860

1.4 Paraxial Diffraction of Narrow Bandwidth Polychromatic

Radiation through Time-Independent Apertures .............869

2. Space-time FOURIER Structure of Scalar Optical Signals ..........876

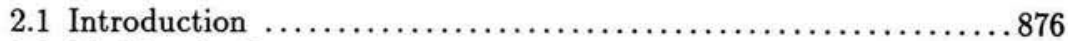

2.2 Multimode Beams ................................ 877

2.3 FOURIER Processing of Scalar Optical Signals ............. 898

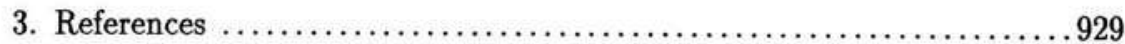

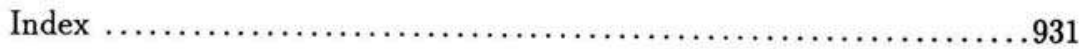

